ISSN 1112-9867

Available online at

http://www.jfas.info

\title{
PARAMETER ESTIMATION FOR FM SIGNALS IN TWO STAGES: NON UNIFORM FAST FOURIER TRANSFORM (NUFFT) AND SHORT-TIME FREQUENCY TRANSFORM
}

\author{
K. Heydari ${ }^{1, *}$, P. Azmi ${ }^{2}$, B. Abbasi ${ }^{2}$ and A. Heydari ${ }^{3}$ \\ ${ }^{1}$ Department of Electrical Engineering, Tarbiat Modares University, Tehran \\ ${ }^{2}$ Faculty of Electrical Engineering, Tarbiat Modares University \\ ${ }^{3}$ Department of Electrical Engineering Shahid Beheshti University, Tehran
}

Published online: 09 June 2016

\begin{abstract}
This study deals with estimating the parameters of the received signal of the poly phase in three orders. The process of estimation occurs in two stages: in the first stage, the high orders of the signal are estimated highly accurately using the Non Uniform FFT. The received signal passes through the componential match filter which has been estimated highly accurately. As it passes through this filter, the order of the ploy phase decreases. In the second stage, the components of the low order of the ploy phase signal are estimated highly accurately using the Short Time Frequency Transform (STFT) method. Finally, the findings of the stimulation in these two stages indicated the efficiency of the presented algorithm for the received signal parameters including the initial frequency, the chirp rate, and the change chirp rate.
\end{abstract}

Keywords: estimation; FM signal; chirp rate; change chirp rate; SFTF; NUFFT.

Author Correspondence, e-mail: k.haydari@gmail.com

doi: http://dx.doi.org/10.4314/jfas.8vi2s.62 


\section{INTRODUCTION}

This paper deals with the analysis as well as the estimation of the parameters of chirp signals with constant amplitude. This type of signal is raised in many applications of signal processing, one of the most important being the radar problem. For instance, consider a radar illuminating a target. Then, the transmitted signal will be affected by two different phenomena. First, it will undergo a phase shift induced by the distance and relative motion between the target and the receiver. Assuming this motion is continuous and differentiable, the phase shift can be adequately modeled as $\Phi(t)=a_{0}+a_{1} t+a_{2} t^{2}+a_{3} t^{3}$, where the $a_{1}, a_{2}$ and $a_{3}$ are either related to speed and acceleration or range and speed, depending on what the radar is intended for and on the kind of waveforms transmitted [1].

Many algorithms have been presented to estimate the received ploy phase signal parameters in many articles. Among various approximate maximum likelihood (ML) sinusoidal parameter estimators [1-4], quadratic interpolation of magnitude peaks in a zero-padded Fast Fourier Transform (FFT) [5] has been widely used due to its simplicity and accuracy. Although it works as an approximate ML estimator for well-separated sinusoids with a large zero-padding factor, its accuracy in practice is restricted by the choice of its design parameters, such as window type, FFT length and zero-padding factor. In order to estimate the initial frequency and the chirp rate parameters, many algorithms have been proposed, and they generally fall into two categories: correlation algorithms and non-correlation algorithms. The correlation algorithms include the higher order ambiguity function [6], cubic phase (CP) function [7-8], higher-order ambiguity function-integrated cubic phase function [9], product generalized cubic phase function [10], and parameters estimation algorithm based on nonuniformly spaced signal sample method [11]. The maximum likelihood method [12], and the modified discrete chirp Fourier transform algorithm for CPS [13] belong to non-correlation algorithms. Correlation algorithms have lower computational cost $\left(O\left(N \log _{2} N\right)\right.$ $\operatorname{or} O\left(N^{2} \log _{2} N\right)$, where $N$ is the effective signal length, but suffers from poor performance in the low SNRs. Compared with the correlation algorithms, non-correlation algorithms are just the opposite, with higher performance, but higher computational load $\left(O\left(N^{3} \log _{2} N\right)\right)$. It is worth noting that all these algorithms above cannot achieve a trade-off between computational cost and anti-noise performance. 


\section{SIGNAL MODEL}

In this section we present the received signal model. To summarize, the model to be considered here is given by

$y(n)=A_{0} \exp \left(i\left(a_{0}+a_{1}(n T)+\frac{a_{2}}{2}(n T)^{2}+\frac{a_{3}}{6}(n T)^{3}\right)\right)+w(n) ; \quad n=-\frac{N}{2}, \ldots ., 0, \ldots ., \frac{N}{2}-1$

Where $w(n)$ denotes additive noise, and $A_{0}$ is the constant amplitude. The Fourier transform (FT) can be represented as

$$
\begin{aligned}
X(n) & =\sum_{n=-\frac{N}{2}}^{\frac{N}{2}-1} A_{0} \exp \left(i\left(a_{0}+a_{1}(n T)+\frac{a_{2}}{2}(n T)^{2}+\frac{a_{3}}{6}(n T)^{3}\right)\right) e^{-j 2 \pi f(n T)}+w(n) \\
& =\sum_{n=-\frac{N}{2}}^{\frac{N}{2}-1} A_{0} \exp \left(i\left(a_{0}+\left(a_{1}-f\right)(n T)+\frac{a_{2}}{2}(n T)^{2}+\frac{a_{3}}{6}(n T)^{3}\right)\right)+\mathrm{w}(\mathrm{n})
\end{aligned}
$$

Where $X(n)$ is Fourier Transform of signal, $A_{0}$ is the constant amplitude, $N$ is an odd integer representing the number of samples, $T$ is the sampling interval, $w(n)$ is the additive complex white Gaussian noise with a variance of $\sigma_{w}$, and $a_{1}, a_{2}$ and $a_{3}$ denote the centroid frequency, chirp rate and its change rate, respectively. The input signal to noise ratio (SNR) can be obtained as

$S N R_{i n}=\frac{\left|A_{0} \exp \left(i\left(a_{1}(n T)+\frac{a_{2}}{2}(n T)^{2}+\frac{a_{3}}{6}(n T)^{3}\right)\right)\right|^{2}}{\sigma_{w}^{2}}=\frac{A_{0}^{2}}{\sigma_{w}^{2}}$

\subsection{The relation between signal length with chirp rate and change rate of chirp rate}

In this section, we attempt to relate signal length with chirp rate and change chirp rate. The result, after using the Fourier Transform, can be represented as

$$
\begin{aligned}
& X_{1}(f)=\sum_{n=-\frac{N}{2}}^{\frac{N}{2}-1} A_{0} \exp \left(i\left(\left(a_{1}-f\right)(n T)+\frac{a_{2}}{2}(n T)^{2}\right)\right)+w(n) \\
& X_{2}(f)=\sum_{n=-\frac{N}{2}}^{\frac{N}{2}-1} A_{0} \exp \left(i\left(\left(a_{1}-f\right)(n T)+\frac{a_{3}}{6}(n T)^{3}\right)\right)+w(n)
\end{aligned}
$$


In order to perceive the relationship between the chirp rate and the change chirp rate and the length of the signal more, the impact of each one of them on the length of the signal is taken into consideration individually. To do so, we investigated the relationship between the length of the signal and the FFT output using equations (4) and (5), and ultimately the relationship between the length of the signal and the FFT output will be obtained through the optimization of equation (6):

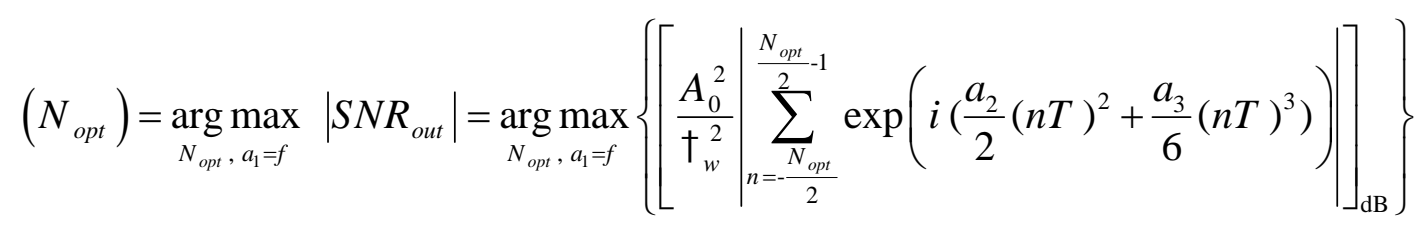

Figure 1 (left) shows the maximum Fourier Transform with different signal lengths and the Figure 1 (right) shows power spectral density of $\mathrm{FT}^{-1}\left\{X_{1}(f)\right\}$ when compensated initial frequency and change chirp rate and Figure 2 (left) shows the maximum Fourier Transform with different signal lengths and Figure 2 (right) shows power spectral density of FT $^{-1}\left\{X_{2}(f)\right\}$ when compensated initial frequency and change chirp rate and parameters are: $a_{2}=60 \mathrm{~Hz} / \mathrm{s}$, and $\mathrm{T}=\frac{1}{600} \mathrm{~s}$. In those figures, we select several representative signal length and $\mathrm{FT}^{-1}\left\{X_{i}(f), i \in\{1,2\}\right\}$.
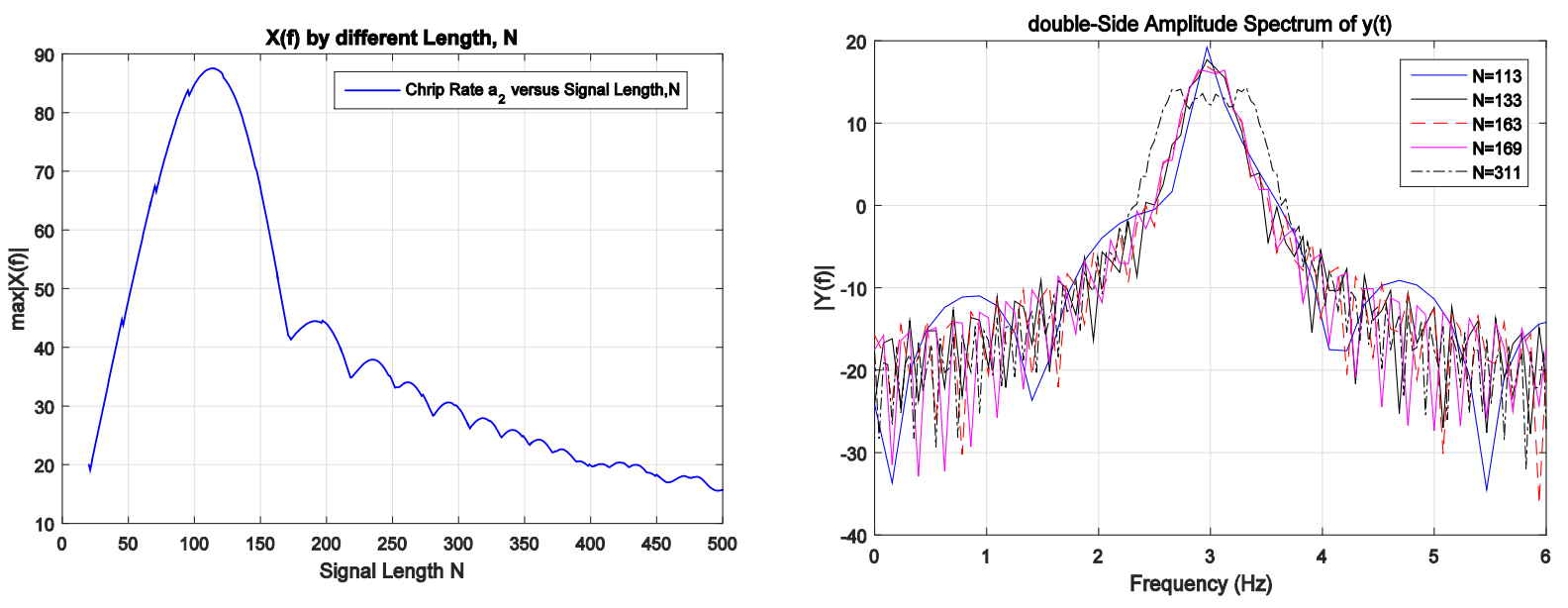

Fig.1. The maximum amount of the signal Fourier transform y(n) with the compensated effect of the initial frequency and the change chirp rate (on the right) and the Power spectral density of signal according to signal length, $\mathrm{N}$, with the compensated the effect of the initial frequency and the change chirp rate (on the left) 

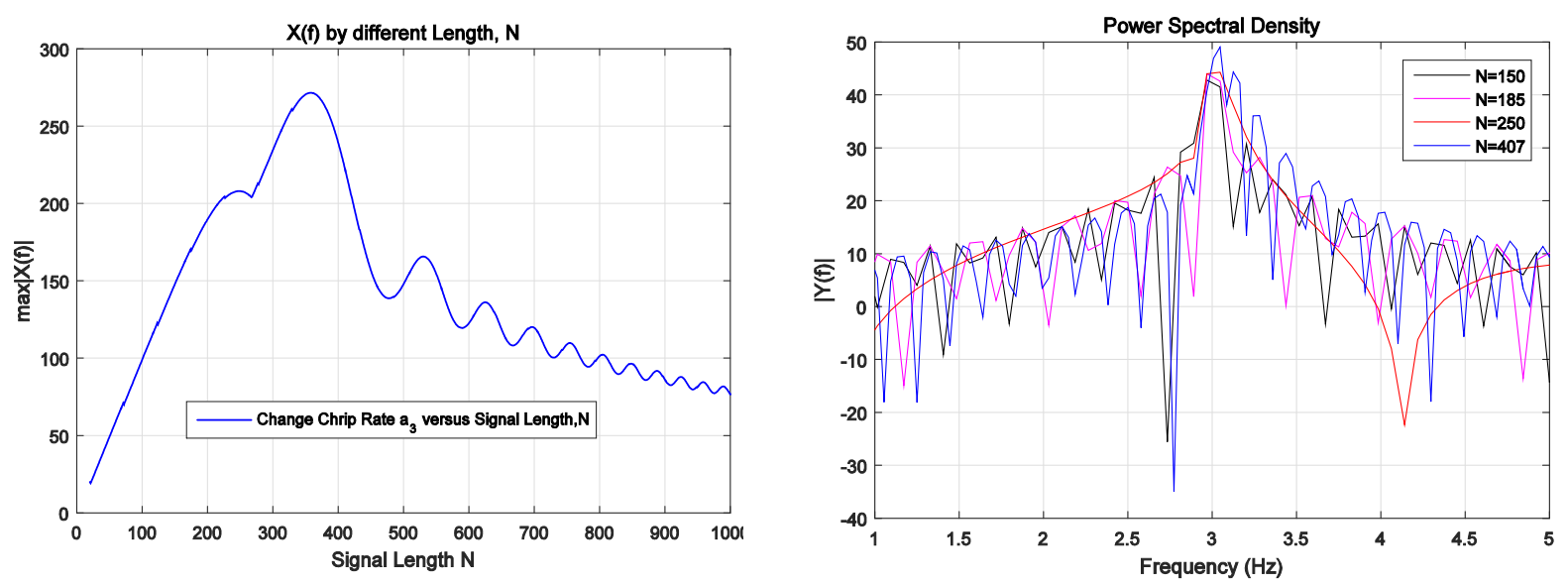

Fig.2. The maximum amount of the signal Fourier transform y(n) with the compensated effect of the initial frequency and the change chirp rate (on the right) and the spectrum signal with the compensate in the effect of the initial frequency and the chirp rate (on the left)

As it can be seen from the above Figures, the optimal amounts of the length of the signal to the chirp rate and change chirp rate have been determined separately. However, if we take these two into account, it will be difficult to determine the optimal amount of the length of the signal. According to [14], the relationship between the length of the signal, chirp rate, and change chirp rate can be determined as follows:

$$
\begin{aligned}
& {\left[N_{1, \text { opt }}\right]_{\mathrm{dB}}=-\frac{\left[a_{2}\right]_{\mathrm{dB}}}{2}+30.1} \\
& {\left[N_{2, o p t}\right]_{\mathrm{dB}}=-\frac{\left[a_{3}\right]_{\mathrm{dB}}}{3}+31.4}
\end{aligned}
$$

Therefore, in order to obtain the amounts of $a_{2}$ and $a_{3}$, we used the above searching stages; in other words, in the first stage of estimation, the following searching stages are used to find the amount of $a_{2}$. By so doing, the complexity of its implementation can be reduced by adjusting the search stage. By considering the computational cost and result of energy accumulation, we perform NUFFT corresponding to $a_{3}$. The change chirp component and its initial frequency are at first reduced using the following cost function.

$$
\left(\hat{a}_{1 \text { coarse }}, \hat{a}_{2 \text { coarse }}, \hat{a}_{3}\right)=\underset{\theta_{1}, \theta_{2}}{\arg \max }\left\{\mid N U F F T_{(n T)^{3}}\left[y(n) \exp \left(-2 i\left(\theta_{1}(n T)+\frac{\theta_{2}}{2}(n T)^{2}\right)\right)\right]\right\}
$$

As it can be seen from the above equation, we need two dimensional searches that increase the complexity and the implementation time of the algorithm. Also, in the above equation 
where $\operatorname{NUFFT}_{(n T)^{3}}$ denotes NUFFT operator with respect to $(n T)^{3}$. Hence, by estimating $a_{3}$, we can define a match filter as follows:

$$
\operatorname{RMF}(n)=\exp \left(-j \frac{\hat{a}_{3}}{6}(n T)^{3}-j \frac{\hat{a}_{2}}{3}(n T)^{2}-j \hat{a}_{1}(n T)\right)
$$

Where in the above terms is the change chirp rate. In case the match filter of one of the components is achieved, we can either remove that component from the received signal or reduce its influence a lot. Hence, upon passing through the match filter, the signal will be as follows:

$$
\begin{aligned}
S(n)= & \mathrm{y}^{*} \mathrm{RMF} \\
= & {\left[A_{0} \exp \left(j\left(a_{0}+a_{1}(n T)+\frac{a_{2}}{2}(n T)^{2}+\frac{a_{3}}{6}(n T)^{3}\right)\right)+w(n)\right] } \\
& \quad \times \exp \left(-j \frac{\hat{a}_{3}}{6}(n T)^{3}-j \frac{\hat{a}_{2}}{3}(n T)^{2}-j \hat{a}_{1}(n T)\right) \\
= & A^{\prime} \exp \left(j\left(\beta(n T)+\frac{\gamma}{2}(n T)^{2}\right)\right)+w^{\prime}(n)
\end{aligned}
$$

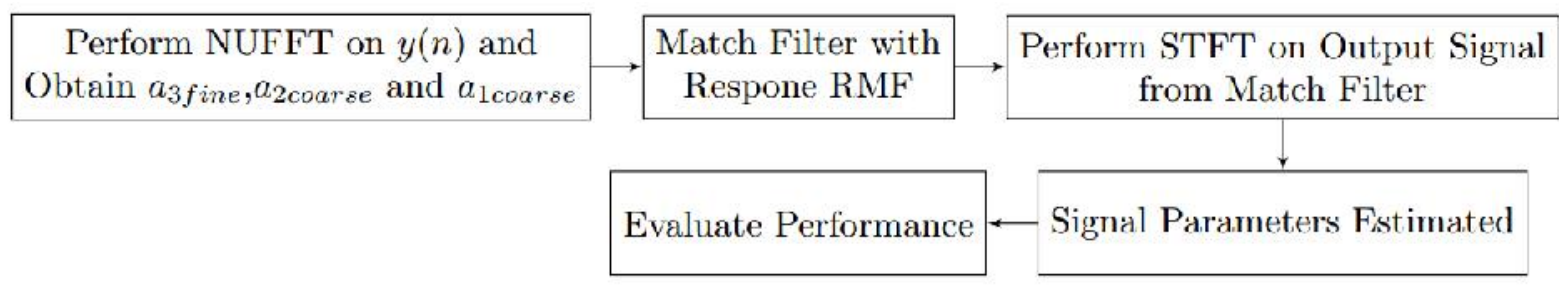

Fig.3. Step of signal parameter estimation by the proposed method

Where in the above equation, $\beta=a_{1}-a_{\text {1coarse }}, \gamma=a_{2}-a_{2 \text { coarse }}, \mathrm{A}^{\prime}=\mathrm{A}_{0} \exp \left(j(n T)^{3}\left(\frac{a_{3}-a_{3 \text { fine }}}{6}\right)\right)$ is considered as fixed amount. Finally, by $w^{\prime}=w \times \mathrm{RMF}$ the multiple of the noise in the response match filter has been achieved. So, if we estimate one of the expressed parameters we can reduce the difficulty of estimation of other parameters. As it was mentioned earlier, the estimation methods of those received signals that have chirp rate can be classified into two groups: associate or formal and informal. The STFT is used to find the initial frequency and the chirp rate. To gain so, the STFT method is implemented on the signal obtained from the stated match filter. So, the analysis framework is based on the uniform-rate short-time Fourier transform (STFT). Hereafter, the centered form of the Fourier transform (FT) is used 
$\operatorname{STFT}\left(t_{m}, w_{k}, h\right)=\sum_{n=-\frac{L-1}{2}}^{n=\frac{L-1}{2}} y\left(\tau_{n}+t_{m}\right) h\left(\tau_{n}\right) \exp \left(-j \tau_{n} w_{k}\right)$

where $h$ is the analysis window, $L$ is the sample size of the Fourier transform, $F_{s}$ is the sampling frequency, $k$ is the frequency bin, and $\tau_{n} \triangleq n / F_{s}$ is the time in seconds of the corresponding sample number $n$. The time corresponding to the center of the STFT window is noted $t_{m}=m / F_{s}$. Finally, $w_{k} \triangleq 2 \pi k F_{s} / N$ is the frequency of the bin $k$. The signal processing based on the STFT relies on the definition of the local model $y\left(t_{m}+\tau\right)=y_{L}(\tau)$. This model is assumed to be valid in the neighborhood of $t_{m}$, and in particular, on the analysis interval centered in $t_{m}$, with a length $W$. This interval usually includes a few overlapping frames $(W>N)$. Then, the output signal obtained from the match filter of the previous stage is investigated by the STFT method which has better estimation of the initial frequency and the chirp rate. To do so, we take the signal obtained from the match filter as follows:

$$
\mathrm{y}_{L}(\tau) \triangleq A^{\prime} \exp \left(\beta \tau+\frac{\gamma \tau^{2}}{2}\right)+w^{\prime}
$$

Where $\beta$ and $\gamma$ are, respectively, the phase, frequency, and frequency change rate for the time $t_{m}$. To emphasize that the amplitude and the chirp rate and initial frequency are constant within the analysis window, the $M$ has been dropped. For the estimation of $\beta$ and $\gamma$, the estimation method was used as it has good performance. Perform STFT on real chirp signal $\mathrm{y}_{L}(\tau)$, the spectrum of which is symmetry about $f=0$. Define the spectrum corresponding to the positive frequency as $\operatorname{PF}(t, f)[15]$ :

$$
\operatorname{PF}(t, f)=\left.\operatorname{SPEC}_{r}(t, f)\right|_{f>0}=\frac{2 \pi}{\sqrt{\xi^{2}+(2 \pi k)^{2}}} \cdot \exp \left\{-\frac{[2 \pi(f-(\beta+\gamma t))]^{2}}{\xi^{2}+(2 \pi k \xi)^{2}}\right\}_{\mid f>0}
$$

The spectrum is the square product of the absolute value of $\operatorname{STFT}_{S}(t, f)$, which is defined as

$$
\left.\operatorname{SPEC}_{r}(t, f)\right|_{f>0}=\left|\operatorname{STFT}_{S}(t, f)\right|^{2}
$$

Using the maximum spectrum on the Sloping line in the (f-t) space, the initial frequency and chirp rate are estimated. The estimated values are obtained as follows [15]: 
$f_{t_{i}}=\arg (\underbrace{\max }_{f=0: F_{s} / 2} \operatorname{PF}\left(t_{i}, \mathrm{f}\right))=\hat{\beta}+\hat{\gamma t}+\varepsilon$

So using the above equation, the initial frequency, $\widehat{\beta}$, and chirp rate, $\hat{\gamma}$ can be obtained. In term $\varepsilon$, in the above equation, shows the error that depends on the estimated frequency.

\section{SIMULATION}

In this section we describe the simulation using the theory in the previous section on MATLAB software. The simulation will be raised in two stages for analysis. In the first stage, the change chirp rate is estimated using the NUFFT method with high precision. Also in this stage, the amounts of the initial frequency and chirp rate are estimated with low accuracy. In the second stage, the estimated parameters of the initial frequency and chirp rate are estimated using NUFFT Method. In simulation parameters the following are set: $A_{0}=1, a_{1}=700, a_{2}=150, a_{3}=78$. Also, this simulation sampling rate equals $\mathrm{F}_{s}=10 \mathrm{KHz}$.

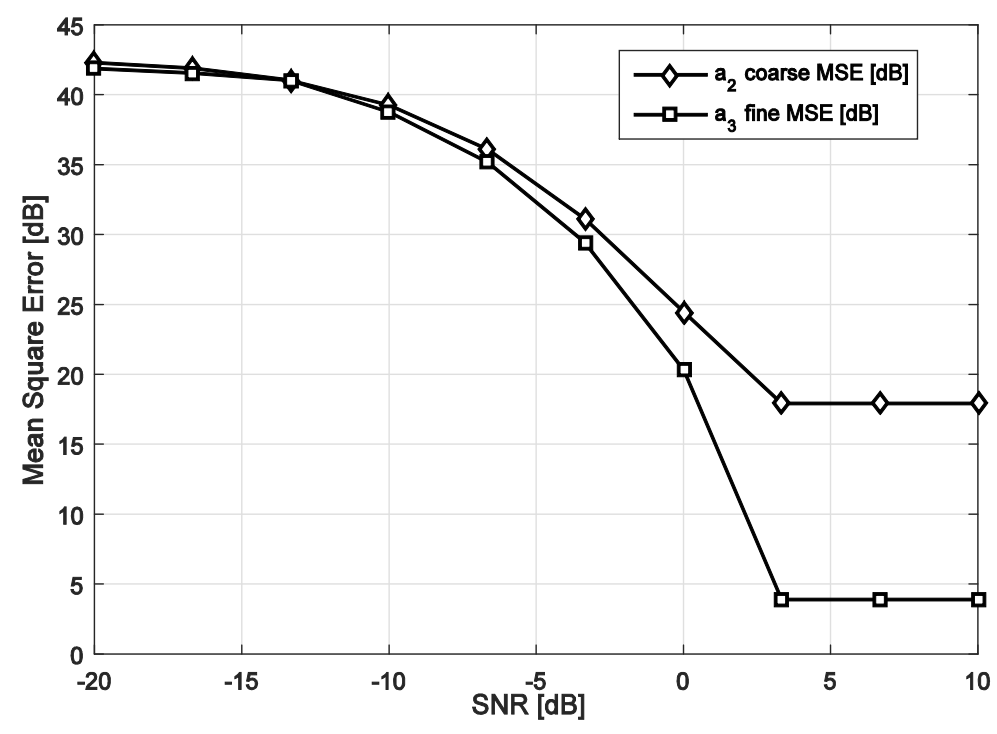

Fig.4. Mean square error from estimation of NUFFT step for chirp rate and change chirp rate

Figure 4 shows the estimation mean square error in step NUFFT and this Figure shows simulations of the estimation error according to SNR. The estimates performance will improve as the SNR increases. As SNR decreases, the performance of estimation method decreases as well. In the presented Figure, the mean square error values are obtained for the chirp rate and change chirp rate. At this stage, the chirp rate component is estimated to be inaccurate; in other words, the coarse values are obtained for chirp rate whereas the change 
chirp rate component is estimated with high accuracy and this description is evident from the Figure. By estimating the change chirp rate component, the matched filter based on estimation can implemented. By implementation of the matched filter, the effect of this component can be compensated and this makes it easier to estimate other parameters.

Figure 5 illustrates the simulation STFT stage. This is also expressed by the estimation Root Mean Square Error at this point to different component according to SNR. As it can be seen in this Figure, if SNR increases, the estimation error has low values and that the performance of the estimation increases. In the other case, if SNR decreases, the performance of estimation decreases too. Another very important note is that in low SNR, RMSE has low values.

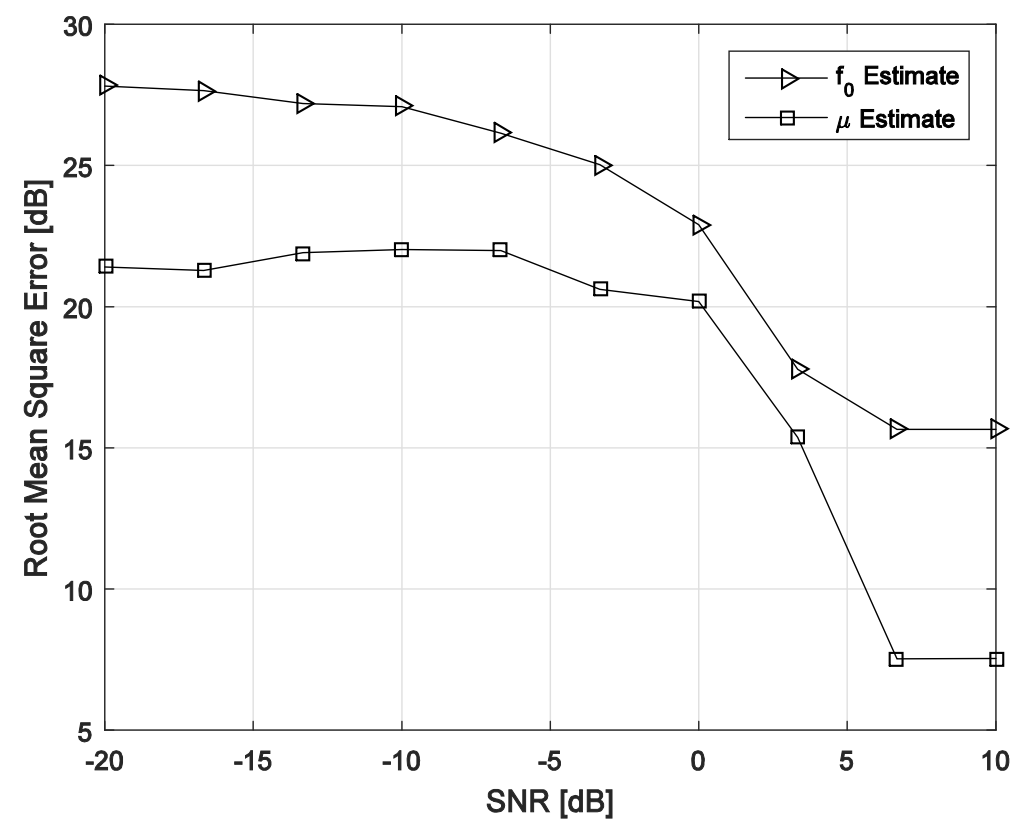

Fig.5. Root mean square error (RMSE) from chirp rate and initial frequency estimation

\section{CONCLUSION}

This paper deals with estimating the parameters of the received signal of the poly phase in three orders. The process of estimation occurs in two stages: in the first stage, the high orders of the signal were estimated highly accurately using the Non Uniform FFT. The received signal passes through the componential match filter which has been estimated highly accurately. As it passes through this filter, the order of the ploy phase decreases. In the second stage, the components of the low order of the ploy phase signal are estimated highly accurately using the Short Time Frequency Transform (STFT) method. Finally, the findings of the stimulation in these two stages indicated the 
efficiency of the presented algorithm for the received signal parameters including the initial frequency, the chirp rate, and the change chirp rate.

\section{REFERENCES}

[1] Rihaczek W., Principles of High-Resolution Radar. New York:McGraw-Hill, 1969.

[2] Kumaresan R and Verma S. On estimating the parameters of chirp signals using rank reduction techniques, in Proc. 21st Asilomar Conf., Pacific Grove, CA, 1987, pp. 555558.

[3] Djuric P and Kay S. M. Parameter estimation of chirp signals, IEEE Trans. Acoust., Speech, Signal Processing, vol. 38, pp. 2118-2126, Dec. 1990.

[4] Peleg $\mathrm{S}$ and Porat B. Linear FM signal parameter estimation from discrete-time observations, IEEE Trans. Aerosp. Electron. Syst., vol. 27, pp. 607-616, July 1991.

[5] S. Peleg and B. Porat, Estimation and classification of polynomialphase signals, IEEE Trans. Inform. Theory, vol. 37, pp. 422-430, Mar. 1991.

[6] Peleg, S. and Porat P. Linear FM signal parameter estimation from discrete-time observations, IEEE Trans. Aerosp. Electron. Syst., Vol. 27, No. 4, 607-616, Jul. 1991.

[7] O'Shea, P., A new technique for instantaneous frequency rate estimation, IEEE Signal Process. Lett., Vol. 9, No. 8, 251-252, Aug. 2002.

[8] O'Shea, P., A fast algorithm for estimating the parameters of a quadratic FM signal, IEEE Trans. Signal Process., Vol. 52, No. 2, 385-393, Feb. 2004.

[9] Wang, Y. and Jiang Y. C. ISAR imaging of a ship target using product high order matched-phase transform, IEEE Geosci. Remote Sens. Lett. , Vol. 6, No. 4, 658-661, Jun. 2009.

[10] Wang, Y., Inverse synthetic aperture radar imaging of manoeuvring target based on range-instantaneous-Doppler and range-instantaneous-chirp-rate algorithms, IET Radar, Sonar and Navigation, Vol. 6, No. 9, 921-928, Jul. 2012.

[11]Wang, Y. and Jiang Y. Inverse synthetic aperture radar imaging of manoeuvring target based on the product generalized cubic phase function, IEEE Geosci. Remote Sens. Lett., Vol. 8, No. 5, 958-962, Sep. 2011.

[12] O'Shea, P. Improving polynomial phase parameter estimation by using nonuniformly spaced signal sample method, IEEE Trans. Signal Process., Vol. 60, No. 7, 3405-3414, Jul. 2012. 
[13]Li, Y., Wu R. Xing M and Bao Z. Inverse synthetic aperture radar imaging of ship target with complex motion, IET Radar, Sonar and Navigation, Vol. 2, No. 6, 395-403, Feb. 2008 .

[14]Jibin, Zheng. Tao Su, Qing Huo Liu, Long Zhang, and Wentao Zhu, Fast Parameter Estimation Algorithm for Cubic Phase Signal Based on Quantifying Effects of Doppler Frequency Shift, Progress In Electromagnetics Research, Vol. 142, 57-74, 2013.

[15] Wang JinZhen, Su ShaoYing and Chen ZengPing., Parameter estimation of chirp signal under low SNR. Science China Information Sciences, February 2015, Vol. 58 020307:1020307:13.

[16]Porat B. Digital Processing of Random Signals: Theory \& Methods. Englewood Cliffs, NJ: Prentice-Hall, 1994.

[17]Englewood Cliffs, NJ: Prentice-Hall, 1994. S. Peleg and B. Friedlander, The discrete polynomial-phase transform, IEEE Trans. Signal Processing, vol. 43, pp. 1901-1914, Aug. 1995.

[18]Dwyer R. F. Fourth-order spectra of Gaussian amplitude modulated sinusoids, J. Acoust. Soc. Amer., vol. 90, pp. 918-926, Aug. 1991.

[19] Swami A. Multiplicative noise models: Parameter estimation using cumulants, Signal Process. , vol. 36, pp. 355-373, Apr. 1994.

[20]Zhou G and Giannakis G. B. On estimating random amplitude modulated harmonics using higher-order spectra, IEEE J. Oceanic Eng., vol. 19, pp. 529-539, Oct. 1994.

[21]Zhou G and Giannakis G. B. Harmonics in multiplicative and additive noise: Performance analysis of cyclic estimators. IEEE Trans. Signal Processing, vol. 43, pp. 1445-1460, June 1995.

[22]Besson $\mathrm{O}$ and Stoica $\mathrm{P}$. Sinusoidal signals with random amplitude: Least-squares estimators and their statistical analysis, IEEE Trans. Signal Processing, vol. 43, pp. 27332744, Nov. 1995.

\section{How to cite this article:}

Heydari K, Azmi P, Abbasi B and Heydari A. Parameter estimation for fm signals in two stages: non uniform fast Fourier transform (NUFFT) and short-time frequency transform. J. Fundam. Appl. Sci., 2016, 8(2S), 467-477. 